\title{
Lipid-Ethanol Interaction Studied by NMR on Bicelles
}

\author{
Bernd W. Koenig* ${ }^{* \dagger}$ and Klaus Gawrisch ${ }^{\ddagger}$ \\ Structural Biology Institute, IBI-2, Research Center Jülich, D-52425 Jülich, Germany, Physical Biology \\ Institute, Heinrich-Heine-Universität Düsseldorf, D-40225 Düsseldorf, Germany, and Laboratory of Membrane \\ Biochemistry and Biophysics, NIAAA, National Institutes of Health, Bethesda, Maryland 20892
}

Received: November 3, 2004; In Final Form: February 8, 2005

\begin{abstract}
The interaction of ethanol with phospholipids was studied in bicelles at a physiologically relevant ethanol concentration of $20 \mathrm{mM}$ and a lipid content of $14 \mathrm{wt} \%$ by high-resolution NMR. Transient association of ethanol with magnetically aligned bicelles imparts a small degree of anisotropy to the solute. This anisotropy allows detection of residual ${ }^{1} \mathrm{H}-{ }^{1} \mathrm{H}$ and ${ }^{1} \mathrm{H}-{ }^{13} \mathrm{C}$ dipolar couplings, which are superimposed on scalar couplings. Residual ${ }^{2} \mathrm{H}$ NMR quadrupole splittings of isotope-labeled ethanol were measured as well. The analysis of residual tensorial interactions yielded information on the orientation and motions of ethanol in the membranebound state. The fraction of phosphatidylcholine-bound ethanol was determined independently by gas chromatography and NMR. About $4 \%$ of ethanol is bound to phosphatidylcholine at a bicelle concentration of $14 \mathrm{wt} \%$ at $40{ }^{\circ} \mathrm{C}$. Free and bound ethanol are in rapid exchange. The lifetime of ethanol association with phosphatidylcholine membranes is of the order of a few nanoseconds.
\end{abstract}

\section{Introduction}

There has been controversy over the amount of membranebound ethanol under physiological conditions. Early MRI experiments conducted in the 1980s suggested that the concentration of free ethanol in the brain is only $(23 \pm 5) \%$ of the blood alcohol concentration, hinting at a large fraction of ethanol bound to brain tissue. ${ }^{1}$ Contrary to those observations there is another school of thought, which assumes that essentially all ethanol is dissolved in bodily fluids. ${ }^{2}$

In the 1990s MRI experiments with improved pulse sequences resulted in ethanol resonances of higher intensity. Still, in some tissues the apparent concentration of free ethanol was significantly lower than expected from blood ethanol content (see Spielman et al. ${ }^{3}$ and references therein). Those differences could be a true reflection of differences in ethanol content of various tissues. It was suggested that a fraction of ethanol may become invisible in the NMR spectra because ethanol immobilization at membrane surfaces dramatically broadens resonance lines and reduces signal intensity due to $\mathrm{T}_{2}$ relaxation. Such "invisibility" requires slow exchange between ethanol in solution and membrane-bound ethanol. Alternatively, an apparently reduced concentration of free ethanol could have resulted also from uncertainties in the calibration procedures of signal intensity.

We established by solid-state ${ }^{2} \mathrm{H} \mathrm{NMR}^{4}$ as well as by ${ }^{1} \mathrm{H}$ magic-angle spinning (MAS) nuclear Overhauser enhancement spectroscopy (NOESY) NMR ${ }^{5,6}$ on lipid membranes that ethanol molecules interact preferentially with the lipid water interface. However, for technical reasons those experiments were conducted at reduced water content that could have driven the ethanol molecules toward the bilayer. There is the need for a new approach that permits investigation of membrane-ethanol

* To whom correspondence should be addressed. Mail: IBI-2/NMR; Forschungszentrum Jülich; D-52425 Jülich; Germany. Tel: +49 2461 615385. Fax: +49 2461 618766. E-mail: b.koenig@fz-juelich.de.

$\dagger$ Structural Biology Institute, IBI-2, Research Center Jülich, and Physical Biology Institute, Heinrich-Heine-Universität Düsseldorf.

$\doteqdot$ National Institutes of Health. interaction at physiologically relevant concentrations of ethanol in the presence of excess water, similar to the situation encountered in human tissue samples.

In the current study, a rapid method is presented that allows characterization of the structure and dynamics of phospholipidbound ethanol by liquid-state NMR. The method is suitable for the investigation of lipid-ethanol interaction at physiologically relevant ethanol concentrations in the range of $1-100 \mathrm{mM}$. Experiments have been conducted on bicelles, i.e., sheets of perforated lipid bilayers that orient spontaneously in the strong magnetic fields of NMR magnets. ${ }^{7,8}$ Solute molecules that associate temporarily with the oriented bilayers have, on average, a small degree of spatial anisotropy in their orientation that is easily detectable as small dipolar couplings or quadrupolar splittings. The high density of pores in the bilayers results in fast equilibration of solute concentrations between compartments, yielding NMR spectra with excellent resolution.

Dipolar couplings from small molecules embedded in anisotropic liquid crystalline media were extensively used for structure elucidation of the solute in the 1970s and 1980s. The sole purpose of the liquid crystals in those studies was to impose an anisotropic component on the rotational diffusion of the solute which prevents the collapse of dipolar couplings that would otherwise result from isotropic averaging. The degree of alignment, and thus the magnitude of the measured dipolar couplings, scales with the concentration of the liquid crystalline medium. Use of highly concentrated liquid crystalline media resulted in large effective dipolar couplings of the solute which translated into complex multiplet patterns of higher order that are difficult to analyze. ${ }^{9}$

We have investigated weak interactions of ethanol with lipid at high water content. Specific interaction of ethanol with the liquid crystalline lipid matrix was deliberately chosen as the means of solute alignment. Therefore, the measured dipolar couplings report on the lipid-bound ethanol conformation. Conditions were chosen such that the induced splittings are small, about 1000-fold less than the static values expected for 
perfectly aligned solute molecules. At those conditions the firstorder character of the NMR spectra is conserved, and spectra are analyzed with ease. This approach has been successfully used in structure determination of soluble proteins and nucleic acids by high-resolution NMR. ${ }^{10}$ The weak dipolar interactions from partial orientation of molecules are referred to as residual dipolar couplings (RDCs). Use of ${ }^{13} \mathrm{C}$ isotope-labeled ethanol in this study enabled determination of eight RDCs of lipid-bound ethanol. Signs of most of the RDCs were deduced from the dependence of the measured couplings on the lipid concentration which governs the degree of ethanol alignment.

\section{Materials and Methods}

Sample Preparation. The phospholipids 1,2-dimyristoyl-snglycero-3-phosphocholine (DMPC) and 1,2-dihexanoyl-sn-glycero-3-phosphocholine (DHPC) were purchased as lyophilized powders from Avanti Polar Lipids (Alabaster, $\mathrm{AL}$ ). Isotopelabeled ethanol ${ }^{13} \mathrm{C}_{2} \mathrm{H}_{5} \mathrm{OH}$ and $\mathrm{C}_{2}{ }^{2} \mathrm{H}_{5} \mathrm{OH},{ }^{2} \mathrm{H}_{2} \mathrm{O}$, and deuteriumdepleted water were from Cambridge Isotope Laboratories (Andover, MA).

A bicelle stock solution containing 15 wt \% lipid was prepared by dissolving appropriate amounts of dry DHPC and DMPC in phosphate buffer $\left(10 \mathrm{mM} \mathrm{KH}_{2} \mathrm{PO}_{4}, 0.1 \mathrm{mM} \mathrm{NaN}\right.$, in $93 \% \mathrm{H}_{2} \mathrm{O} / 7 \%{ }^{2} \mathrm{H}_{2} \mathrm{O}$ or in $100 \%{ }^{2} \mathrm{H}$-depleted water, $\mathrm{pH}$ adjusted to 6.1 ) following a standard protocol. ${ }^{11}$ The molar ratio $q$ of DMPC/DHPC was 3:1. The ethanol stock solution and phosphate buffer were added to the bicelle stock to achieve the intended sample composition. Samples with 20 and $80 \mathrm{mM}$ ethanol in $(5 \pm 1)$ and $(14 \pm 1)$ wt $\%$ bicelle solution were prepared.

Small unilamellar DMPC vesicles were obtained by mixing DMPC with phosphate buffer and ethanol stock to establish concentrations of $5 \mathrm{wt} \%$ lipid and $20 \mathrm{mM}$ ethanol. The lipid suspension was subjected to three rounds of annealing by cycling the temperature between 45 and $0{ }^{\circ} \mathrm{C}$ followed by sonication for $3 \times 1 \mathrm{~min}$ at $40^{\circ} \mathrm{C}$ with a Branson 250 sonifier equipped with a microtip.

Partially hydrated large multilamellar DMPC vesicles were prepared by gravimetrically adding ethanol solution in deuteriumdepleted water to the lipid dried in vacuum. Sealed samples were mixed to apparent homogeneity by centrifugation. Details of the procedure have been published previously. ${ }^{4}$

Bicelle samples and DMPC liposomes for ${ }^{31} \mathrm{P}$ NMR measurements contained $10 \mathrm{mM}$ Tris $-\mathrm{HCl}$ instead of $10 \mathrm{mM} \mathrm{KH}_{2} \mathrm{PO}_{4}$ in the buffer.

NMR Measurements. High-resolution one-dimensional ${ }^{1} \mathrm{H}$, ${ }^{13} \mathrm{C}$, and ${ }^{2} \mathrm{H}$ NMR spectra were recorded on Bruker DMX600 and DRX800 spectrometers. Triple resonance $(\mathrm{HCN})$ probes equipped with a pulsed field gradient along the $z$-axis were used. Both ${ }^{13} \mathrm{C}$ coupled and GARP-decoupled ${ }^{1} \mathrm{H}$ NMR spectra were acquired. Water suppression was achieved by a hard pulse 1-1 excitation sequence ${ }^{12}$ followed by a 1-1 refocusing pulse ${ }^{13}$ that was flanked by two field gradients for dephasing the residual water signal. ${ }^{13} \mathrm{C}$ NMR spectra were recorded both with and without ${ }^{1} \mathrm{H}$ decoupling during acquisition, respectively. ${ }^{2} \mathrm{H}$ NMR spectra on bicelle samples were recorded using the deuterium lock channel of the HCN-probe.

${ }^{2} \mathrm{H}$ NMR powder spectra of partially hydrated DMPC/ $\mathrm{C}_{2}{ }^{2} \mathrm{H}_{5}-$ $\mathrm{OH} / \mathrm{H}_{2} \mathrm{O}$ samples (molar ratio 1:1:10) were recorded with a quadrupolar echo sequence ${ }^{14}$ on a Bruker DMX300 spectrometer at $43{ }^{\circ} \mathrm{C}$. A stationary dual resonance probe with a 4-mm solenoid sample coil was used.

${ }^{31} \mathrm{P}$ NMR spectra were recorded on a Varian Unity INOVA 600 spectrometer at a resonance frequency of $243 \mathrm{MHz}$ using a dual resonance probe. GARP ${ }^{1} \mathrm{H}$ decoupling was applied during acquisition. Samples contained $14 \mathrm{wt} \%$ lipid, and spectra were recorded at $40{ }^{\circ} \mathrm{C}$. The chemical shift scale was calibrated using a capillary filled with 4 vol $\% \mathrm{H}_{3} \mathrm{PO}_{4}$ as an external standard. ${ }^{31} \mathrm{P}$ NMR spectra were referenced to the isotropic signal of $2.5 \mathrm{wt} \%$ DHPC in buffer that was set to $0 \mathrm{ppm}$.

Anisotropic spectra of ethanol $(20 \mathrm{mM})$ were observed in the presence of 5 and $14 \mathrm{wt} \%$ bicelles at $40{ }^{\circ} \mathrm{C}$. Three complete sets of ${ }^{1} \mathrm{H}$ and ${ }^{13} \mathrm{C}$ NMR spectra were recorded on the DMX600 for each bicelle concentration over a period of 2 months. Samples were stored at $6{ }^{\circ} \mathrm{C}$ between measurements. One complete set of anisotropic spectra of $20 \mathrm{mM}$ ethanol was recorded on the DRX800 for both bicelle concentrations. In addition, two complete sets of ${ }^{1} \mathrm{H}$ and ${ }^{13} \mathrm{C}$ NMR spectra were measured for $80 \mathrm{mM}$ ethanol in a $5 \mathrm{wt} \%$ bicelle solution at 40 ${ }^{\circ} \mathrm{C}$ using the DMX600. Deuterium NMR spectra were recorded for $20 \mathrm{mM} \mathrm{C}_{2}{ }^{2} \mathrm{H}_{5} \mathrm{OH}$ in a $14 \mathrm{wt} \%$ bicelle solution at $40{ }^{\circ} \mathrm{C}$ at the DMX600. Error estimates on the couplings are based on the scatter of the multiplet splittings observed at a given sample composition.

Isotropic ${ }^{1} \mathrm{H}$ and ${ }^{13} \mathrm{C}$ NMR spectra of ethanol were recorded in water, in a dispersion of small unilamellar DMPC vesicles, and in an isotropic bicelle solution. Subscripts $\alpha$ and $\beta$ are used in this manuscript to refer to the methylene $\left(\mathrm{C}_{\alpha}, \mathrm{H}_{\alpha 1}, \mathrm{H}_{\alpha 2}\right)$ and methyl group $\left(\mathrm{C}_{\beta}, \mathrm{H}_{\beta 1}, \mathrm{H}_{\beta 2}, \mathrm{H}_{\beta 3}\right)$ of ethanol, respectively.

Order Parameters from Residual Dipolar Couplings. The static dipolar coupling for any given pair of spin $1 / 2$ nuclei $\mathrm{P}$ and $\mathrm{Q}$ is given by

$$
D_{\mathrm{PQ}}^{0}=-\left(\frac{\mu_{0}}{4 \pi}\right) \frac{h}{2 \pi} \frac{\gamma_{\mathrm{P}} \gamma_{\mathrm{Q}}}{r_{\mathrm{PQ}}{ }^{3}} \frac{1}{2 \pi}
$$

Here $\gamma_{\mathrm{P}}$ and $\gamma_{\mathrm{Q}}$ are the gyromagnetic ratios of nuclei $\mathrm{P}$ and $\mathrm{Q}, \quad r_{\mathrm{PQ}}$ is the internuclear distance, $-\mu_{0}$ is the magnetic permittivity of vacuum, and $h$ is Planck's constant. Internuclear distances used in this manuscript are based on ethanol coordinates published by Kadzhar et al. ${ }^{15} D_{\mathrm{PQ}}^{0}$ applies to a static internuclear vector PQ aligned parallel to the direction of the magnetic field $\mathbf{B}_{0}$. Dynamic motion of the vector PQ reduces the observed residual dipolar coupling, $D_{\mathrm{PQ}}^{\exp }$, according to

$$
D_{\mathrm{PQ}}^{\exp }=D_{\mathrm{PQ}}^{0}\left\langle\frac{3 \cos ^{2} \theta-1}{2}\right\rangle=D_{\mathrm{PQ}}^{0} S_{\mathrm{PQ}}^{\exp }
$$

The angle $\theta$ specifies the instantaneous orientation of PQ with respect to $\mathbf{B}_{0}$. Brackets indicate a time average. The bracketed term defines the order parameter $S_{\mathrm{PQ}}^{\text {exp }}$ of vector PQ.

Residual dipolar couplings are deduced from comparison of ethanol spectra in anisotropic vs isotropic environments. Multiplet patterns in the first-order spectra of ethanol recorded in isotropic media reflect the isotropic component of the scalar coupling, $J_{\mathrm{PQ}}$. Scalar couplings are not observed between the methyl protons ( $\mathrm{A}_{3}$ system) and between the methylene protons $\left(\mathrm{A}_{2}\right.$ system) of ethanol due to magnetic equivalence. In anisotropic media the multiplet splitting of a weakly coupled spin pair (AX) amounts to $J_{\mathrm{AX}}+2 D_{\mathrm{AX}}$ instead of $J_{\mathrm{AX}}$, where $D_{\mathrm{AX}}$ is the residual dipolar coupling between spins A and X. Here it is assumed that the anisotropic component of the scalar coupling is negligibly small. ${ }^{16,17}$ Protons of an $\mathrm{A}_{n}$ system usually lose their magnetic equivalence if subjected to an asymmetric environment and give rise to a multiplet with multiplicity $n$ and a splitting of $3 D_{\mathrm{AA}}$.

Order Parameters from Quadrupolar Splittings. The order parameter of a carbon-deuterium bond, $S_{\mathrm{CD}}$, in uniaxially 
oriented molecules is related to the measured quadrupolar splitting, $\Delta v_{\mathrm{Q}}$, according to

$$
\Delta v_{\mathrm{Q}}=\frac{3}{2}\left(\frac{e^{2} q Q}{h}\right)\left|S_{\mathrm{CD}}\right|
$$

Here $\left(e^{2} q Q / h\right)$ is the static quadrupolar coupling constant which amounts to $\sim 168 \mathrm{kHz}$ for methyl and methylene segments. ${ }^{18}$ The splitting of the peak doublet in a first-order ${ }^{2} \mathrm{H}$ NMR spectrum reflects only the magnitude but not the sign of the quadrupolar coupling interaction. Therefore, only the magnitude of $S_{\mathrm{CD}}$ is obtained from eq 3 .

Ethanol Partitioning by Gas Chromatography. Samples were prepared by weighing an appropriate amount of ethanolcontaining bicelle mixture into headspace vials. Control vials contained an identical volume of ethanol solution without the lipid. Vials were sealed and put through seven repetitive cycles of freezing, thawing, and vortexing to ensure homogeneous distribution. Measurements of ethanol concentration in the headspace were performed using a HP 6890 series gas chromatograph $(\mathrm{GC})$ equipped with a flame ionization detector. A $30 \mathrm{~m} \times 0.53 \mathrm{~mm} \times 40 \mu \mathrm{m}$ HP-PLOT Q polystyrene-divinylbenzene-based column was utilized in order to resolve ethanol from various short-chain hydrocarbon gases that can arise from lipid oxidation. A HP 7694 headspace sampler with a 1-mL sample loop and silicosteel transfer line was connected directly to the split inlet of the GC (all Hewlett-Packard, Wilmington, DE). Operating conditions were as follows: headspace sampler oven, loop, and transfer line temperatures were 50, 80, and 80 ${ }^{\circ} \mathrm{C}$, respectively; time parameters for vial equilibration, vial pressurization, loop fill, loop equilibration, injection time, and GC cycle time were $60,0.15,0.15,0.15,0.5$, and $10 \mathrm{~min}$, respectively. Because no internal standard was used, additional precautions were required to ensure reproducibility of headspace injection volume. This was achieved through insulation of exposed areas between sampler and GC inlet to minimize temperature gradients, maintaining an isothermal oven temperature of $140^{\circ} \mathrm{C}$, and preconditioning the system with injections of similar ethanol concentration for at least $6 \mathrm{~h}$ prior to running samples in order to achieve steady-state conditions. Headspace vials were continuously shaken during equilibration and pressurized with 22.0 psi of helium. The inlet temperature was maintained at $250{ }^{\circ} \mathrm{C}$ with a split ratio of $2: 1$. Helium was used as the carrier gas with a flow rate of $6.0 \mathrm{~mL} / \mathrm{min}$. Data acquisition and peak integration were performed using HP Chemstation software (Hewlett-Packard).

\section{Results}

${ }^{1} \mathrm{H}$ and ${ }^{13} \mathrm{C}$ NMR Data. First-order isotropic ${ }^{1} \mathrm{H}$ and ${ }^{13} \mathrm{C}$ NMR spectra of ethanol were obtained in water, in a $5 \mathrm{wt} \%$ dispersion of ultrasonicated small unilamellar DMPC vesicles at $40{ }^{\circ} \mathrm{C}$, and in a 5 wt $\%$ bicelle solution at $15^{\circ} \mathrm{C}$. The absolute values of the isotropic scalar couplings obtained in these three environments are identical within experimental error and are listed in Table 1 . The one and three bond $J$ couplings measured are known to be positive. ${ }^{19}$ The ${ }^{2} J_{\mathrm{CH}}$ couplings between $\mathrm{C}_{\beta}$ and $\mathrm{H}_{\alpha}$ and between $\mathrm{C}_{\alpha}$ and $\mathrm{H}_{\beta}$ are most likely negative based on comparison with ${ }^{2} J_{\mathrm{CH}}$ couplings of ethane derivatives. ${ }^{20}$

Anisotropic ethanol spectra were recorded in 5 and 14 wt \% bicelles at $40{ }^{\circ} \mathrm{C}$. Couplings between nonequivalent nuclei are again first order; however the size of the multiplet splittings has changed to $J_{\mathrm{AX}}+2 D_{\mathrm{AX}}$ (Figure 1). Both the high $\mathbf{B}_{0}$ field strength and the rather low degree of ethanol alignment are responsible for preservation of first-order characteristics. The
TABLE 1: Data on Couplings of ${ }^{13} \mathrm{C}$ and ${ }^{1} \mathrm{H}$ Nuclei of Ethanol

\begin{tabular}{cccccc}
\hline $\begin{array}{c}\text { interacting } \\
\text { nuclei P, Q }\end{array}$ & $\begin{array}{c}J_{\mathrm{PQ}}^{\text {iso }} \\
{[\mathrm{Hz}]}\end{array}$ & $\begin{array}{c}D_{\mathrm{PQ}}^{0} \\
{[\mathrm{~Hz}]}\end{array}$ & $\begin{array}{c}D_{\mathrm{PQ}}^{\exp } \\
{[\mathrm{Hz}]}\end{array}$ & $\begin{array}{c}D_{\mathrm{PO}}^{\text {calc }} \\
{[\mathrm{Hz}]}\end{array}$ & \multicolumn{1}{c}{$S_{\mathrm{PQ}}^{\exp }\left[10^{-4}\right]$} \\
\hline $\mathrm{C}_{\alpha} \mathrm{C}_{\beta}$ & +37.3 & -2147 & $+(0.5 \pm 0.1)$ & +0.5 & $-(2.3 \pm 0.5)$ \\
$\mathrm{C}_{\alpha} \mathrm{H}_{\alpha}$ & +143.0 & -23146 & $-(31.4 \pm 1)$ & -31.7 & $+(13.6 \pm 0.5)$ \\
$\mathrm{C}_{\beta} \mathrm{H}_{\beta}$ & +125.7 & -22732 & $-(2.1 \pm 0.1)$ & -1.9 & $+(0.9 \pm 0.05)$ \\
$\mathrm{C}_{\alpha} \mathrm{H}_{\beta}$ & $(-) 4.9$ & -3014 & $(+)(0.6 \pm 0.1)$ & +0.5 & $(-)(2.0 \pm 0.3)$ \\
$\mathrm{C}_{\beta} \mathrm{H}_{\alpha}$ & $(-) 2.5$ & -2972 & $(-)(2.6 \pm 0.1)$ & -2.6 & $(+)(8.7 \pm 0.3)$ \\
$\mathrm{H}_{\alpha} \mathrm{H}_{\beta}$ & +7.2 & & $-(2.5 \pm 0.1)$ & -2.6 & \\
$\mathrm{H}_{\alpha} \mathrm{H}_{\alpha}$ & 0 & -21465 & $( \pm)(34.3 \pm 1)$ & -31.2 & $( \pm)(16.0 \pm 0.4)$ \\
$\mathrm{H}_{\beta} \mathrm{H}_{\beta}$ & 0 & -21371 & $( \pm)(2.7 \pm 0.1)$ & -2.7 & $( \pm)(1.26 \pm 0.05)$
\end{tabular}

${ }^{a}$ Isotropic scalar couplings at $40{ }^{\circ} \mathrm{C}$, signs in parentheses are indirectly determined. ${ }^{b}$ Residual dipolar couplings in $14 \mathrm{wt} \%$ bicelles at $40{ }^{\circ} \mathrm{C}$.

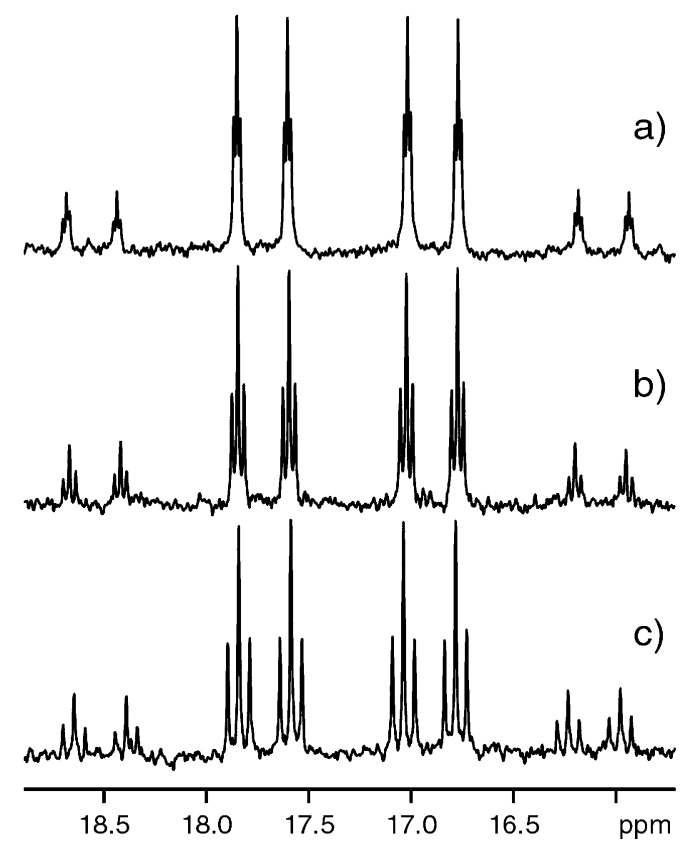

Figure 1. Methyl region of first-order ${ }^{13} \mathrm{C}$ NMR spectra of ${ }^{13} \mathrm{C}_{2} \mathrm{H}_{5} \mathrm{OH}$ recorded without proton decoupling at $40{ }^{\circ} \mathrm{C}$. Ethanol was studied in water (a), in $5 \mathrm{wt} \%$ bicelles (b), and in $14 \mathrm{wt} \%$ bicelles (c). Apparent changes in multiplet splittings reflect residual dipolar couplings as a result of partial alignment of ethanol, which increases with bicelle concentration.

absence of second-order effects greatly simplifies interpretation of spectra but also prevents deduction of the relative sign of the couplings from the spectra. Both the $\mathrm{CH}_{3}$ and $\mathrm{CH}_{2}$ protons of ethanol become nonequivalent in the bicelle environment and give rise to a triplet and a doublet in the ${ }^{1} \mathrm{H}$ NMR spectra, respectively, with multiplet splittings equal to $3 D_{\mathrm{AA}}$. The entire set of eight residual dipolar couplings, $D_{\mathrm{PQ}}^{\mathrm{exp}}$, observed in the presence of $14 \mathrm{wt} \%$ bicelles at $40{ }^{\circ} \mathrm{C}$ are given in Table 1 .

The anisotropy is caused by fast exchange of ethanol between an isotropically tumbling form in bulk solution and a motionally restricted form temporarily associated with the magnetically aligned membrane surface of the bicelle. Under conditions of fast exchange, dipolar couplings which originate from the bound state are reduced by a uniform factor. At our experimental conditions, this factor ranges from 25 to 75 . All observed splittings show a linear dependence on bicelle concentration (Figure 2). This linear behavior allows determination of the sign of the residual dipolar coupling, provided the sign of the corresponding $J$-coupling is known. Data analysis yields four dipolar couplings with sign, two dipolar couplings for which the sign is almost certainly known (signs given in parentheses), and two dipolar couplings with unknown sign (cf. Table 1). 

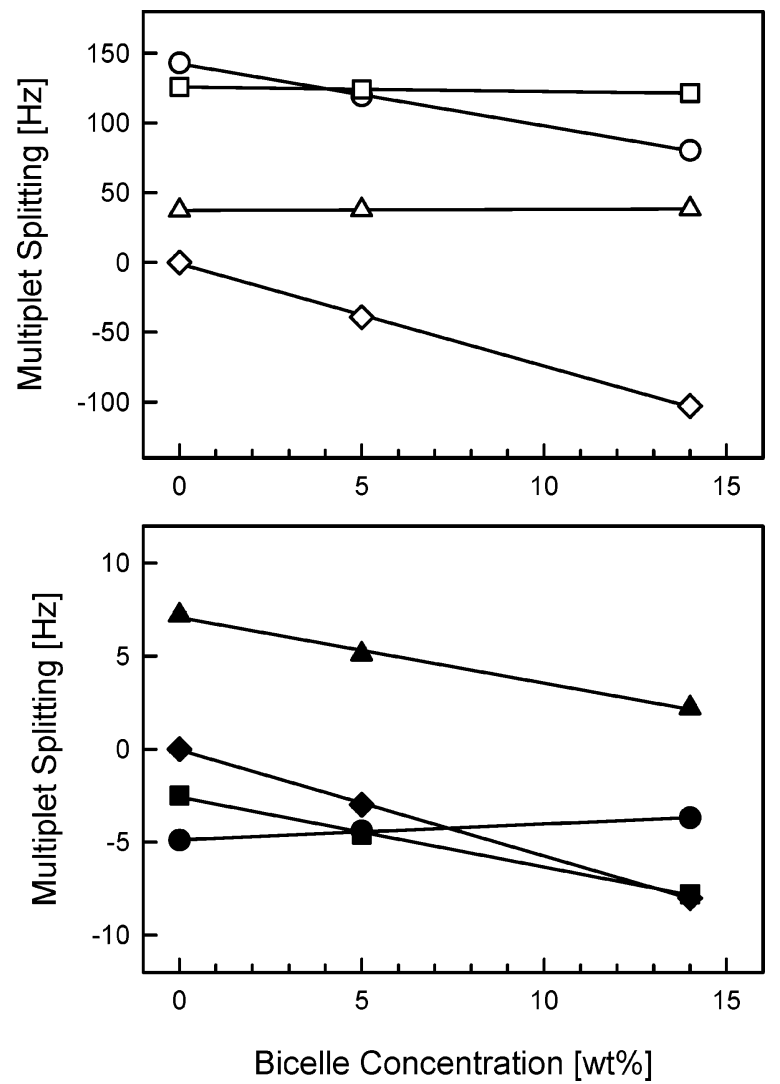

Figure 2. Dependence of observed multiplet splittings on bicelle concentration for $\mathrm{C}_{\alpha} \mathrm{H}_{\alpha}$ (open circle), $\mathrm{C}_{\beta} \mathrm{H}_{\beta}$ (open square), $\mathrm{C}_{\alpha} \mathrm{C}_{\beta}$ (open triangle), $\mathrm{H}_{\alpha} \mathrm{H}_{\alpha}$ (open diamond), $\mathrm{C}_{\alpha} \mathrm{H}_{\beta}$ (solid circle), $\mathrm{C}_{\beta} \mathrm{H}_{\alpha}$ (solid square), $\mathrm{H}_{\beta} \mathrm{H}_{\alpha}$ (solid triangle), and $\mathrm{H}_{\beta} \mathrm{H}_{\beta}$ (solid diamond) interactions. Splittings in isotropic samples (0 wt \% bicelles) represent the scalar coupling. The sign of the $J$ couplings was either known a priori or derived during data analysis. Splittings in anisotropic samples are the sum of the scalar and a specific multiple of the dipolar coupling (see text for details). Clearly there is a linear correlation between splittings and bicelle concentration; lines shown represent linear regression of the data. The sign of the slope of the regression line provides the sign of the dipolar coupling.

Table 1 also shows static dipolar coupling constants, $D_{\mathrm{PQ}}^{0}$, calculated from eq 1 and the derived order parameters $S_{\mathrm{PQ}}^{\exp }$ according to eq 2 .

${ }^{2}$ H NMR Data. ${ }^{2} \mathrm{H}$ NMR spectra of deuterated ethanol in a 14 wt \% bicelle solution show three superimposed doublets that were assigned to the $\mathrm{C}^{2} \mathrm{H}_{3}$ and $\mathrm{C}^{2} \mathrm{H}_{2}$ signals of ethanol and to trace amounts of ${ }^{2} \mathrm{HHO}$. The ethanol signals have fine structure due to small ${ }^{2} \mathrm{H}-{ }^{2} \mathrm{H}$ dipolar and scalar couplings. Quadrupolar splittings of 333.8 and $20.7 \mathrm{~Hz}$ were deduced for the $\mathrm{C}^{2} \mathrm{H}_{2}$ and $\mathrm{C}^{2} \mathrm{H}_{3}$ groups, respectively, which translate into order parameters of $13.2 \times 10^{-4}$ and $0.8 \times 10^{-4}$ for the corresponding $\mathrm{C}-{ }^{2} \mathrm{H}$ bond vectors according to eq 3 . As expected, these $S_{\mathrm{CD}}$ order parameters agree very well with the $S_{\mathrm{CH}}$ order parameters for the same bond vectors derived from residual dipolar couplings (cf. Table 1).

Order Parameter Analysis. One objective was to analyze residual dipolar couplings in terms of reorientational motion of lipid-bound ethanol molecules. However, order parameters listed in Table 1 reflect both reorientational and internal motions of the bound solute as well as the exchange of ethanol between the free and lipid-bound states. Analysis is restricted to the $\mathrm{C}_{\beta} \mathrm{H}_{3}-\mathrm{C}_{\alpha} \mathrm{H}_{2}$ fragment of ethanol for which order parameters have been determined. Internal motions in ethanol, such as small-amplitude vibrations and fast axial rotation of the methyl group about the $\mathrm{C}-\mathrm{C}$ bond, occur on a time scale of roughly
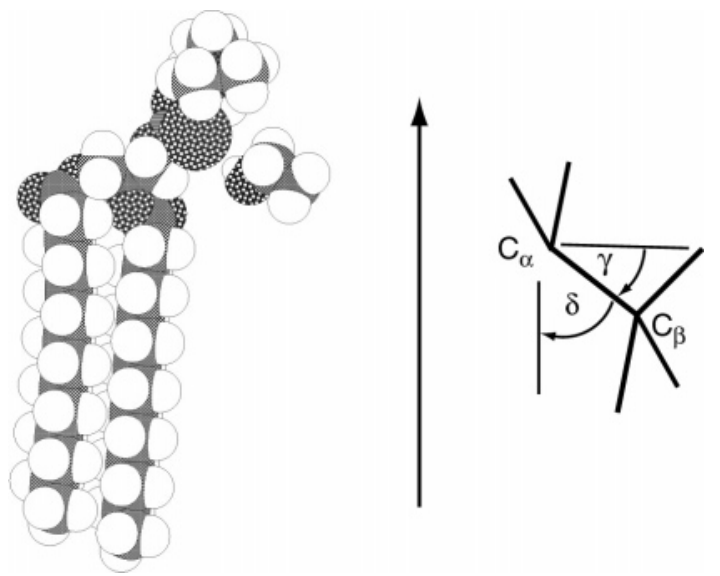

Figure 3. Schematic representation of relative orientation of lipid and ethanol during temporary association (left). The average orientation of the $\mathrm{C}_{\alpha}-\mathrm{C}_{\beta}$ bond of ethanol relative to the membrane normal (represented by the arrow) was found to be $51.2^{\circ}$. A stick representation of the $\mathrm{C}_{\alpha} \mathrm{H}_{2} \mathrm{C}_{\beta} \mathrm{H}_{3}$ fragment of ethanol is shown on the right. Angles $\gamma$ and $\delta$ are used in eqs 4 and 5 to describe methyl proton rotation about the $\mathrm{C}_{\alpha}-\mathrm{C}_{\beta}$ bond and rotation of the $\mathrm{C}_{\alpha} \mathrm{H}_{2} \mathrm{C}_{\beta}$ fragment about the membrane normal, respectively. In the figure, $\gamma$ denotes the angle between $\mathrm{C}_{\alpha}-\mathrm{C}_{\beta}$ and $\mathrm{C}_{\alpha}-\mathrm{H}_{\beta}$ internuclear vectors and $\delta$ is the angle between the membrane normal and the $\mathrm{C}_{\alpha}-\mathrm{C}_{\beta}$ bond vector.

$10^{-12}$ to $10^{-14} \mathrm{~s}$, while the reorientation of ethanol in the lipidbound state is dominated by the rotation of the lipid about the normal of the liquid crystalline membrane occurring on a time scale of $10^{-9} \mathrm{~s}$ (in combination with slow lateral diffusion and wobble of the lipid). Finally, lifetimes of ethanol in the free and bound state, respectively, are on the order of $10^{-7}$ to $10^{-9}$ s. Observation of just one set of averaged dipolar couplings indicates that also the exchange process is fast compared to the effective dipolar couplings in the lipid-bound state.

Fast internal motions and reorientation are very likely uncorrelated given the large difference in time scales. In principle, separation of small-amplitude vibrations from slow uncorrelated motions is possible by replacing the distance term $r_{\mathrm{PQ}}{ }^{-3}$ in eq 1 by the vibrationally averaged quantity $\left\langle r_{\mathrm{PQ}}{ }^{-3}\right\rangle$. The substitution removes the dependence of the order parameter on small-amplitude vibrations. However, this would require knowledge of the modes and amplitudes of vibrations of lipidbound ethanol in water. In lieu of such information, intramolecular distances $r_{\mathrm{PQ}}$ in the current analysis are based on the gas-phase structure of the trans form of ethanol determined by radio spectroscopy ${ }^{15}$ assuming they present a reasonable approximation of vibrationally averaged intramolecular distances. Small-amplitude vibrations usually account for at most a few percent of the reduction in order observed in the liquid crystalline phase, making this approximation rather uncritical.

Fast Axial Rotation of the Terminal Methyl of Ethanol. Rapid rotation of the methyl group on top of the much slower molecular reorientation reduces dipolar couplings involving $\mathrm{H}_{\beta}$ protons in a conformation sensitive manner. The order parameter $S_{\mathrm{C}_{\alpha} \mathrm{C}_{\beta}}$ can be predicted from any of the order parameters $S_{\mathrm{PQ}}^{\exp }$ measured for pairs of nuclei within the $\mathrm{C}_{\beta} \mathrm{H}_{3}-\mathrm{C}_{\alpha}$ fragment, provided this fragment rotates as a rigid rotor about the $\mathrm{C}_{\alpha}-\mathrm{C}_{\beta}$ bond:

$$
S_{\mathrm{C}_{\alpha} \mathrm{C}_{\beta}}=\frac{2 S_{\mathrm{PQ}}^{\exp }}{3 \cos ^{2} \gamma-1}
$$

Here $\gamma$ is the angle between the internuclear vector PQ and the rotation axis $\mathrm{C}_{\alpha}-\mathrm{C}_{\beta}$ (cf. Figure 3 ). The directly determined 
TABLE 2: Order Parameter of the $\mathbf{C}_{\alpha}-\mathbf{C}_{\boldsymbol{\beta}}$ Rotation Axis

\begin{tabular}{cccr}
\hline $\begin{array}{c}\text { interacting } \\
\text { nuclei P, Q }\end{array}$ & $\gamma^{a}(\mathrm{deg})$ & $S_{\mathrm{PQ}}^{\exp }\left[10^{-4}\right]$ & \multicolumn{1}{c}{$S_{\mathrm{C}_{\alpha} \mathrm{C}_{\beta}}\left[10^{-4}\right]$} \\
\hline $\mathrm{C}_{\alpha} \mathrm{C}_{\beta}$ & 0 & $-(2.3 \pm 0.5)$ & $-(2.3 \pm 0.5)$ \\
$\mathrm{C}_{\beta} \mathrm{H}_{\beta}$ & 70.5 & $+(0.9 \pm 0.05)$ & $-(2.8 \pm 0.2)$ \\
$\mathrm{C}_{\alpha} \mathrm{H}_{\beta}$ & 28.7 & $(-)(2.0 \pm 0.3)$ & $(-)(3.0 \pm 0.5)$ \\
$\mathrm{H}_{\beta} \mathrm{H}_{\beta}$ & 90 & $( \pm)(1.26 \pm 0.05)$ & $( \pm)(2.5 \pm 0.1)$
\end{tabular}

${ }^{a}$ Angle between the internuclear vector $r_{\mathrm{PQ}}$ and the $\mathrm{C}_{\alpha}-\mathrm{C}_{\beta}$ axis.

order parameter of the $\mathrm{C}_{\alpha}-\mathrm{C}_{\beta}$ axis agrees within experimental error with order parameters calculated for the same axis based on the measured $\mathrm{C}_{\beta} \mathrm{H}_{\beta}, \mathrm{C}_{\alpha} \mathrm{H}_{\beta}$, and $\mathrm{H}_{\beta} \mathrm{H}_{\beta}$ dipolar couplings (cf. Table 2). Error estimates of the four $S_{\mathrm{C}_{\alpha} \mathrm{C}_{\beta}}$ values obtained are based exclusively on a uniform uncertainty of $\pm 0.1 \mathrm{~Hz}$ of the measured dipolar couplings. The requirement of a unique sign of the four $S_{\mathrm{C}_{\alpha} \mathrm{C}_{\beta}}$ values in Table 2 reconfirms the initial choice of a negative sign of the $\mathrm{C}_{\alpha} \mathrm{H}_{\beta}$ scalar coupling and yields a negative sign for the $\mathrm{H}_{\beta} \mathrm{H}_{\beta}$ dipolar coupling. The weighted average of $S_{\mathrm{C}_{\alpha} \mathrm{C}_{\beta}}$ amounts to $-(2.6 \pm 0.2) \times 10^{-4}$. The good agreement between the four independently obtained order parameters for the $\mathrm{C}_{\alpha}-\mathrm{C}_{\beta}$ axis indicates that the $\mathrm{C}_{\beta} \mathrm{H}_{3}-\mathrm{C}_{\alpha}$ fragment of lipid-bound ethanol can indeed be considered as a rapidly rotating rigid rotor with a geometry that is reasonably well approximated by the trans conformation of gaseous ethanol.

Reorientation of the $\mathbf{C}_{\boldsymbol{\beta}}-\mathbf{C}_{\alpha} \mathbf{H}_{2}$ Fragment. The four order parameters determined for vectors $\mathrm{C}_{\alpha} \mathrm{C}_{\beta}, \mathrm{C}_{\alpha} \mathrm{H}_{\alpha}, \mathrm{C}_{\beta} \mathrm{H}_{\alpha}$, and $\mathrm{H}_{\alpha} \mathrm{H}_{\alpha}$ contain information on the whole-body reorientational motion of the $\mathrm{C}_{\beta}-\mathrm{C}_{\alpha} \mathrm{H}_{2}$ entity. In the following we consider the case of an internally rigid $\mathrm{C}_{\beta}-\mathrm{C}_{\alpha} \mathrm{H}_{2}$ fragment completely lacking any methylene rotation about the $\mathrm{C}_{\alpha}-\mathrm{C}_{\beta}$ axis. The simplest scenario is a lipid-bound ethanol fragment that undergoes uniaxial rotation about an axis $X$. This situation is sketched in Figure 3. The rotation axis $X$ must be parallel to the membrane normal, which is represented by an arrow in Figure 3. If this model applies, then the rigid fragment $\mathrm{C}_{\beta}-\mathrm{C}_{\alpha} \mathrm{H}_{2}$ has a unique order parameter, $S_{X}$, relative to the rotation axis. Further, $S_{X}$ is related to each of the four order parameters $S_{\mathrm{PQ}}^{\exp }$ observed within the rigid $\mathrm{C}_{\beta}-\mathrm{C}_{\alpha} \mathrm{H}_{2}$ fragment according to

$$
S_{X}=\frac{2 S_{\mathrm{PQ}}^{\exp }}{3 \cos ^{2} \delta-1}
$$

where $\delta$ is the angle between the rotation axis and vector PQ. Rotation axis $X$ must be located in the mirror plane of the ethanol molecule containing the $\mathrm{C}_{\beta}, \mathrm{C}_{\alpha}$, and $\mathrm{O}$ atoms given the trans conformation of ethanol. Otherwise, one would expect nonidentical dipolar couplings for $\mathrm{C}_{\alpha} \mathrm{H}_{\alpha 1} / \mathrm{C}_{\alpha} \mathrm{H}_{\alpha 2}$ and $\mathrm{C}_{\beta} \mathrm{H}_{\alpha 1} /$ $\mathrm{C}_{\beta} \mathrm{H}_{\alpha 2}$ which are not observed. To test if an axis $X$ exists so that eq 5 yields identical $S_{X}$ for each of the four $S_{\mathrm{PQ}}^{\exp }$ of the $\mathrm{C}_{\beta}-\mathrm{C}_{\alpha} \mathrm{H}_{2}$ fragment, a computer-based search was conducted varying the angle between the $\mathrm{C}_{\alpha}-\mathrm{C}_{\beta}$ bond and a trial axis $X$ from 0 to $180^{\circ}$. For each trial angle four $S_{X}$ values were calculated according to eq 5 using the measured order parameters of $\mathrm{C}_{\alpha} \mathrm{C}_{\beta}, \mathrm{C}_{\alpha} \mathrm{H}_{\alpha}, \mathrm{C}_{\beta} \mathrm{H}_{\alpha}$, and $\mathrm{H}_{\alpha} \mathrm{H}_{\alpha}$. The search aimed at minimizing the root-mean-square deviation (rmsd) of the four $S_{X}$ values. The smallest rmsd was found for an angle of $51.3^{\circ}$. Although the order parameters $S_{X}$ are very close to each other, the difference between the smallest (based on $\mathrm{C}_{\alpha} \mathrm{H}_{\alpha}$ data) and the largest $S_{X}$ (based on $\mathrm{H}_{\alpha} \mathrm{H}_{\alpha}$ data) does slightly exceed the experimental uncertainty (cf. Table 3 ). However, mutual agreement of all four $S_{X}$ values within experimental error is obtained by changing the $\mathrm{H}_{\alpha}-\mathrm{C}_{\alpha}-\mathrm{H}_{\alpha}$ angle of ethanol from $108.652^{\circ}$ (trans conformation of gaseous ethanol ${ }^{15}$ ) to any value between $103.8^{\circ}$ and $107.1^{\circ}$. The minimum rmsd of the four $S_{X}$ values
TABLE 3: Rotation of the Rigid $\mathrm{C}_{\beta}-\mathrm{C}_{\alpha} \mathrm{H}_{2}$ Fragment about the $X$ Axis

\begin{tabular}{cccr}
\hline $\begin{array}{c}\text { interacting } \\
\text { nuclei P, Q }\end{array}$ & $\delta^{a}(\mathrm{deg})$ & \multicolumn{1}{c}{$S_{\mathrm{PQ}}^{\exp }\left[10^{-4}\right]$} & \multicolumn{1}{c}{$S_{X}\left[10^{-4}\right]$} \\
\hline $\mathrm{C}_{\alpha} \mathrm{C}_{\beta}$ & 51.3 & $-(2.6 \pm 0.2)^{b}$ & $-(30 \pm 2)$ \\
$\mathrm{C}_{\alpha} \mathrm{H}_{\alpha}$ & 81.9 & $13.6 \pm 0.5$ & $-(29 \pm 1)$ \\
$\mathrm{C}_{\beta} \mathrm{H}_{\alpha}$ & 68.4 & $(+) 8.7 \pm 0.3$ & $(-)(30 \pm 1)$ \\
$\mathrm{H}_{\alpha} \mathrm{H}_{\alpha}$ & 90 & $( \pm) 16.0 \pm 0.4$ & $( \pm)(32 \pm 1)$
\end{tabular}

${ }^{a}$ Angle between the internuclear vector $r_{\mathrm{PQ}}$ and the rotation axis $X$. ${ }^{b}$ Weighted average from Table 2.

from their mean value occurs at the $\mathrm{H}_{\alpha}-\mathrm{C}_{\alpha}-\mathrm{H}_{\alpha}$ angle of $105.435^{\circ}$ at an angle of $51.2^{\circ}$ between the rotation axis $X$ and the $\mathrm{C}_{\alpha}-\mathrm{C}_{\beta}$ bond. The small deviation of the $\mathrm{H}_{\alpha}-\mathrm{C}_{\alpha}-\mathrm{H}_{\alpha}$ angle from ideal tetrahedral geometry indicated by our data is not unusual and is most likely caused by hydrogen bonding of the ethanol hydroxyl to the lipid headgroup.

Ethanol Motions Further Reduce the Dipolar Couplings. On top of the two axial rotations about the $\mathrm{C}_{\alpha}-\mathrm{C}_{\beta}$ and the $X$ axis, respectively, which reduce the order parameter of individual bond vectors in a specific manner, there are whole body motions of lipid-bound ethanol that reduce the order parameters of all intramolecular bond vectors by a unique but unknown factor. Such collective motions arise, for example, from the highly dynamic nature of the lipid molecule, from overall bilayer motions, and from fluctuations in the geometry of the predicted hydrogen bond between lipid headgroup and ethanol. Lateral diffusion of lipid-bound ethanol across the highly curved edge regions of the bicelles does also contribute. As a result there is a set of order parameters, $S_{\mathrm{PQ}}^{\text {bound }}$, which reflect the dynamic state of lipid-bound ethanol in the bicelle environment. However, these order parameters of bound ethanol are not directly discernible from the measured RDCs.

The measured order parameters $S_{\mathrm{PQ}}^{\mathrm{exp}}$ listed in Table 1 can be considered as products of the $S_{\mathrm{PQ}}^{\text {bound }}$ values, a factor of $-1 / 2$ accounting for the perpendicular orientation of the membrane normal with respect to the $\mathbf{B}_{0}$ field, and a unique scaling factor, that accounts for the exchange of ethanol between the lipidbound and the isotropically tumbling form in solution. This scaling factor equals the fraction of time that a given ethanol molecule spends in the bound state, which is identical to the fraction of lipid-bound ethanol molecules $\left[\mathrm{E}_{\mathrm{b}}\right] /\left[\mathrm{E}_{\mathrm{t}}\right]$.

$$
S_{\mathrm{PQ}}^{\exp }=-\frac{1}{2} S_{\mathrm{PQ}}^{\text {bound } \frac{\left[\mathrm{E}_{\mathrm{b}}\right]}{\left[\mathrm{E}_{\mathrm{t}}\right]}}
$$

Separation of the two contributions to $S_{\mathrm{PQ}}^{\exp }$ is not possible, unless one of them is determined independently.

Order Parameters of Liposome-Bound Ethanol. Order parameters of ethanol bound to large multilamellar lipid vesicles (MLVs) can be directly measured if all the ethanol present in the lipid dispersion is lipid-bound. Such a situation is most likely encountered in partially dehydrated membranes, where all the water is strongly bound to the lipid headgroup and no free water is available for solvation of ethanol. Barry and Gawrisch have studied the quadrupolar splitting $(\Delta v)_{\perp}$ of $\mathrm{CH}_{3} \mathrm{C}^{2} \mathrm{H}_{2} \mathrm{OH}$ in nonoriented dioleoyl phosphatidylcholine (DOPC) membranes containing only 10 solvent molecules, i.e., water plus ethanol, per lipid. Variation of the ethanol concentration from 0.2 to 2 molecules per lipid produced only a small change of the $(\Delta v)_{\perp}$ of the ethanol methylene group from 9.44 to $6.81 \mathrm{kHz} .{ }^{4}$ This indicates that essentially all ethanol is lipid-bound in this composition range.

We measured quadrupolar splittings $(\Delta v)_{\perp}$ of 8.3 and 0.93 $\mathrm{kHz}$ for the $\mathrm{C}^{2} \mathrm{H}_{2}$ and $\mathrm{C}^{2} \mathrm{H}_{3}$ groups of deuterated ethanol in 
nonoriented dispersions of DMPC at $43{ }^{\circ} \mathrm{C}$ and a lipid/water/ ethanol molecular ratio of 1:10:1. The calculated order parameters $\left|S_{\mathrm{CH}}^{\mathrm{MLV} \text {-bound }}\right|$ for the $\mathrm{C}_{\alpha} \mathrm{H}_{\alpha}$ and $\mathrm{C}_{\beta} \mathrm{H}_{\beta}$ bonds of DMPC MLV-bound ethanol amount to $6.6 \times 10^{-2}$ and $7.4 \times 10^{-3}$, respectively. Please note, an additional factor of $1 / 2$ is required on the right-hand side of eq 3 if $\left|S_{\mathrm{CD}}\right|$ is to be related to the $\left(\Delta v_{\mathrm{Q}}\right)_{\perp}$ component of a ${ }^{2} \mathrm{H}$ NMR powder spectrum.

Order Parameters of Bicelle-Bound Ethanol. In a bicelle system the ethanol can bind to both DMPC and DHPC. Lipid order parameters in a bicelle are reduced relative to the corresponding order parameters in DMPC MLVs, mainly due to overall bilayer motions in excess of those occurring in DMPC MLVs and lateral diffusion of the lipid across the highly curved rim region of the bicelles. ${ }^{7}$ The reduction of lipid order in bicelles can be estimated from the position of the ${ }^{31} \mathrm{P}$ resonances of magnetically aligned bicelles. ${ }^{7,21}$ Order parameters $S_{\text {Bic }}^{\mathrm{DMPC}}$ and $S_{\mathrm{Bic}}^{\mathrm{DHPC}}$ can be defined, which reflect this reduction in order of DMPC and DHPC, respectively, in bicelles as compared to DMPC in MLVs:

$$
S_{\mathrm{Bic}}^{\mathrm{DMPC}}=\frac{\delta_{\mathrm{Bicelle}}^{\mathrm{DMPC}}}{\delta_{90^{\circ}, \mathrm{MLV}}^{\mathrm{DMPC}}} \quad \text { and } \quad S_{\mathrm{Bic}}^{\mathrm{DHPC}}=\frac{\delta_{\mathrm{Bicelle}}^{\mathrm{DHPC}}}{\delta_{90^{\circ}, \mathrm{MLV}}^{\mathrm{DMPC}}}
$$

Here $\delta_{\text {Bicelle }}^{\text {DMPC }}$ and $\delta_{\text {Bicelle }}^{\text {DHPC }}$ are the chemical shifts of the sharp ${ }^{31} \mathrm{P}$ resonances of DMPC and DHPC, respectively, in the magnetically aligned bicelle sample and $\delta_{90^{\circ} \text { MLV }}^{\text {DMPC }}$ is the position of the $90^{\circ}$ component of the ${ }^{31} \mathrm{P}$ CSA powder pattern of DMPC MLVs at the same temperature. The signal intensity in the powder pattern of the 14 wt \% DMPC sample is heavily biased toward the $90^{\circ}$ component due to partial alignment of the DMPC bilayer in the strong magnetic field of $14.1 \mathrm{~T}$. Please note that the chemical shifts in eq 7 are referenced to the isotropic ${ }^{31} \mathrm{P}$ NMR signal of DHPC and DMPC which have the same shift within experimental error and have been set to 0 ppm.

The value of $\delta_{90^{\circ}, \mathrm{MLV}}^{\mathrm{DMPC}}$ was $-15.6 \mathrm{ppm}$ for DMPC MLVs at $40{ }^{\circ} \mathrm{C} ; \delta_{\text {Bicelle }}^{\text {DMPC }}=-8.7 \mathrm{ppm}$ and $\delta_{\text {Bicelle }}^{\text {DHPC }}=-2.9 \mathrm{ppm}$ were observed for 14 wt $\%$ bicelles both in the absence of ethanol and with $20 \mathrm{mM}$ ethanol. At $80 \mathrm{mM}$ ethanol the value of $\delta_{\text {Bicelle }}^{\text {DMPC }}$ shows an insignificant reduction to $-8.6 \mathrm{ppm}$. Accordingly, order parameters $S_{\mathrm{Bic}}^{\mathrm{DMPC}}=0.56$ and $S_{\mathrm{Bic}}^{\mathrm{DHPC}}=0.19$ were obtained for 14 wt $\%$ bicelles at $q=3$ and $40{ }^{\circ} \mathrm{C}$.

The reduction of lipid order in bicelles vs MLVs causes an equivalent reduction of the order parameters of lipid-bound ethanol. The order parameters of bicelle-bound ethanol, $S_{\mathrm{PQ}}^{\text {bound }}$, may be derived from the corresponding order parameters of MLV-bound ethanol, $S_{\mathrm{PQ}}^{\mathrm{MLV}-\text {-bound }}$, if we assume identical binding affinity of ethanol for DMPC and DHPC and fast exchange of ethanol between the free state and the two qualitatively different binding sites on the bicelle on the NMR time scale:

$$
S_{\mathrm{PQ}}^{\text {bound }}=\left(v_{\mathrm{DMPC}} S_{\mathrm{Bic}}^{\mathrm{DMPC}}+v_{\mathrm{DHPC}} S_{\mathrm{Bic}}^{\mathrm{DHPC}}\right) S_{\mathrm{PQ}}^{\mathrm{MLV}-\text { bound }}
$$

where $v_{\text {DMPC }}$ and $v_{\text {DHPC }}$ are the mole fractions of DMPC and DHPC in the lipid bicelle which amount to 0.75 and 0.25 , respectively, at $q=3$. Using the order parameters $S_{\mathrm{PQ}}^{\mathrm{MLV} \text {-bound }}$ of $\mathrm{C}_{\alpha} \mathrm{H}_{\alpha}$ and $\mathrm{C}_{\beta} \mathrm{H}_{\beta}$ reported above, bicelle-bound order parameters $S_{\mathrm{PQ}}^{\text {bound }}$ of 0.031 and 0.0035 are predicted, respectively.

Partition of Ethanol between the Aqueous Phase and a Lipid-Bound Pool. The lipid-bound ethanol fraction $\left[\mathrm{E}_{\mathrm{b}}\right] /\left[\mathrm{E}_{\mathrm{t}}\right]$ is estimated by inserting the experimental order parameters of $\mathrm{C}_{\alpha} \mathrm{H}_{\alpha}$ and $\mathrm{C}_{\beta} \mathrm{H}_{\beta}$ (cf. Table 1) and $S_{\mathrm{PQ}}^{\text {bound }}$ from above into eq 6 , yielding values of 0.088 and 0.051 , respectively.
Independently, ethanol partitioning between bicelles and the water phase was evaluated by gas chromatographic experiments. Lipid water dispersions containing an excess of water are threephase systems in which ethanol in the lipid phase is in thermodynamic equilibrium with ethanol in the free water phase and the gas phase above the sample (headspace). After equilibration the chemical potentials of ethanol in the lipid phase, in the water phase, and in the gas phase are identical. Therefore the ethanol vapor pressure is a precise measure of ethanol concentration in the excess water phase. Compared to an ethanol solution without lipid, we observed a $(4 \pm 1) \%$ decrease of ethanol concentration in the water phase in the presence of 14 wt \% lipid bicelles, indicating that a fraction of 0.04 of ethanol molecules is associated with the lipid phase. This is somewhat lower than the values predicted by ethanol order parameter analysis.

The gas chromatographic analysis of ethanol partitioning into bilayers is more direct than the estimate based on comparison of ethanol order parameters in multilamellar liposomes and bicelle dispersions. The tendency of slightly higher values from the order parameter analysis most likely reflects differences between ethanol binding in multilamellar liposomes and bicelles. The difference between predicted ethanol partitioning based either on $\mathrm{C}_{\alpha} \mathrm{H}_{\alpha}$ or $\mathrm{C}_{\beta} \mathrm{H}_{\beta}$ order parameter analysis, respectively, is another indication that dynamic orderings of lipid-bound ethanol in multilamellar liposomes at reduced water content and high ethanol concentration and in dilute bicelles at low ethanol concentration do not perfectly match.

Binding Strength of Lipid-Ethanol Interaction. Let us assume for simplicity that binding of ethanol to phosphatidylcholines follows the mass action law for a one-to-one complex. If the concentration of lipid and ethanol is well below the dissociation constant $K_{\mathrm{D}}$ of the complex, then the concentration of the free molecules, [lipid $]$ and $\left[\mathrm{E}_{\mathrm{f}}\right]$, can be approximated by the total concentrations, [lipid $\left.{ }_{\mathrm{t}}\right]$ and $\left[\mathrm{E}_{\mathrm{t}}\right]$, yielding the following approximate relation for $K_{\mathrm{D}}$ :

$$
K_{\mathrm{D}}=\frac{\left[\mathrm{E}_{\mathrm{f}}\right]\left[\operatorname{lipid}_{\mathrm{f}}\right]}{\left[\mathrm{E}_{\mathrm{b}}\right]} \approx \frac{\left[\mathrm{E}_{\mathrm{t}}\right]\left[\mathrm{lipid}_{\mathrm{t}}\right]}{\left[\mathrm{E}_{\mathrm{b}}\right]}
$$

The total molar concentration of phosphatidylcholines, i.e., DMPC and DHPC, in a $14 \mathrm{wt} \%$ bicelle solution is $\sim 225 \mathrm{mM}$. Assuming a fraction of lipid-bound ethanol of 0.04 , a $K_{\mathrm{D}}$ of $\sim 5.6 \mathrm{M}$ is obtained. For simplicity it is assumed that binding affinities of ethanol to DMPC and DHPC are identical. Furthermore, the small fraction of monomeric DHPC in the sample, which amounts to a few $\mathrm{mM}$ at most, has been neglected.

Two sets of RDCs were measured in $5 \mathrm{wt} \%$ bicelles at 20 and $80 \mathrm{mM}$ ethanol, respectively. The RDCs in these two sets are identical within experimental error, despite the 4-fold difference in ethanol concentrations. Indeed, invariability of the lipid-bound fraction of ethanol $\left[\mathrm{E}_{\mathrm{b}}\right] /\left[\mathrm{E}_{\mathrm{t}}\right]$, and thus of the measured RDCs, is expected as long as [lipid $t$ and $\left[\mathrm{E}_{\mathrm{t}}\right]$ are well below $K_{\mathrm{D}}$. According to eq 9 and Figure 2, the fraction of phosphatidylcholine-bound ethanol is linear to the lipid concentration under physiological conditions.

The average lifetime of a single binding event of ethanol to phosphatidylcholine lipid can be estimated from $K_{\mathrm{D}}$ if a diffusion-controlled association rate constant, $k_{\text {on }}$, of $\sim 10^{8}$ $\mathrm{M}^{-1} \mathrm{~S}^{-1}$ is assumed:

$$
K_{\mathrm{D}}=\frac{k_{\text {off }}}{k_{\text {on }}}=\frac{1}{\tau_{\mathrm{B}} k_{\text {on }}}
$$


where $k_{\text {off }}=1 / \tau_{\mathrm{B}}$ is the dissociation rate constant of the interaction. An average lifetime of $1.8 \mathrm{~ns}$ is obtained for the temporary phosphatidylcholine-ethanol association.

Comparison of Measured and Calculated RDCs. The measured RDCs can be reproduced based on the geometry of the trans conformation of ethanol, ${ }^{15}$ rapid methyl group rotation, rigid rotation of the $\mathrm{C}_{\beta}-\mathrm{C}_{\alpha} \mathrm{H}_{2}$ fragment about the membrane normal, and a uniform scaling factor that applies to all RDCs. For the calculation of the $\mathrm{H}_{\alpha}-\mathrm{H}_{\beta}$ couplings, rapid methyl rotation was assumed. The uniform scaling factor accounts for whole body motions of lipid-bound ethanol, the exchange of ethanol between the free and lipid-bound state, and the average orientation of the membrane normal with respect to the magnetic field. Using this scaling factor as the only adjustable parameter, we determined the set of $D_{\mathrm{PQ}}^{\text {calc }}$ that most faithfully reproduces the measured RDCs (cf. Table 1). Seven of the eight measured RDCs are very close to the calculated values. The RCD of the $\mathrm{H}_{\alpha}-\mathrm{H}_{\alpha}$ vector deviates by about $10 \%$ from the calculation, most likely due to compromised tetrahedral geometry of the methylene group as discussed above.

\section{Discussion}

${ }^{2} \mathrm{H}$ NMR based order parameter measurements ${ }^{4,22}$ as well as ${ }^{1} \mathrm{H}$ MAS NOESY NMR studies ${ }^{5,6}$ have significantly contributed to the current understanding of lipid-ethanol interactions in membranes. For technical reasons, previous investigations were restricted to lipid dispersions containing only modest amounts of water. Increasing the water-to-lipid ratio beyond 10:1 in nonoriented samples causes inhomogeneous sample morphology that eventually leads to line broadening and noninterpretable NMR spectra. The relatively low number of 10 water molecules per ethanol in those studies raised criticism regarding the high ethanol concentration in the solvent that was way above physiological ethanol concentrations in bodily fluids.

In this study model membranes were used that align spontaneously in strong magnetic fields. ${ }^{7,11}$ Close to perfect magnetic alignment of bicelles, in combination with fast axial rotation of lipid-bound ethanol about the membrane normal, results in a unique set of anisotropic interactions that bear information on orientation and motions of bound ethanol. Bicelles do not compartmentalize the water phase, avoiding inhomogeneous line broadening. Fast exchange between bound and free ethanol reduces anisotropic interactions by a factor that is equal to the bound fraction of ethanol. As a consequence experiments can be conducted in high-resolution mode. The ethanol concentrations of 20 and $80 \mathrm{mM}$ are well within the physiological range. The lipid concentration in the bicelle mixture is comparable to lipid concentrations in brain and other tissues.

The partition of ethanol between the membrane and the excess water phase was determined by headspace gas chromatography, and results were compared with estimates from order parameter analysis. The more direct gas chromatographic analysis yielded ( $4 \pm 1) \%$ bound ethanol, while predictions based on order parameter analysis resulted in somewhat higher values. We assume that this deviation is a reflection of differences in ethanol orientation and motions between the multilamellar liposomes, containing restricted amounts of water and high concentrations of ethanol, and the much more dilute bicelle system.

Magnetically aligned model membranes provide a novel means for investigation of specific interactions between membrane constituents and ethanol or other weakly binding solutes under physiological conditions. For example, the methodology may be applied to study the interaction of membranes with anesthetics or carbohydrates. ${ }^{23}$ Although the current study was conducted on model membranes composed of just two phosphatidylcholine lipids with different hydrocarbon chain length, it is conceivable to prepare bicelles which host a variety of lipids as well as proteins. For example, bicelles tolerate significant amounts of charged lipids, ${ }^{24}$ which would allow investigation of the influence of lipid headgroups on the lipid-ethanol interaction. Asymmetrically shaped membrane fragments with a high density of transmembrane $\alpha$-helices align spontaneously in the NMR magnet and might be suitable for investigation of protein-ethanol interactions. Examples of alignable membranes are purple membrane fragments from bacteria ${ }^{25,26}$ and disk membranes from the retina of vertebras. ${ }^{27}$ It can be envisioned to use RDCs to study weak interactions of ethanol, anesthetics, drugs, and other biologically active substances with membranes, which closely resemble specific regions or tissues in the nervous system. Such studies might reveal or even quantify preferred interactions of biologically active solutes with distinct lipid classes or membrane proteins.

We have demonstrated that a complete set of RDCs is easily extracted from first-order ${ }^{1} \mathrm{H}$ and ${ }^{13} \mathrm{C}$ NMR spectra of ethanol. The RDCs are then used to derive order parameters, including the sign, of intramolecular vectors of the ligand. A subset of these order parameters was also obtained from residual quadrupolar splittings of deuterated ethanol. However, in addition to the smaller number of accessible deuterium order parameters, only their magnitude but not their sign can be measured.

Precise measurement of a large set of RDCs provides important structural information on rigid fragments of the membrane-bound ligand. The current study demonstrates that the lipid-bound $\mathrm{CH}_{3}-\mathrm{CH}_{2}$ fragment of ethanol is reasonably well approximated by the trans conformation of gaseous ethanol, most likely with slightly reduced tetrahedral angles in the methylene segment. The average orientation of lipid-bound ethanol relative to the membrane normal was found to be $51.2^{\circ}$. The distribution of ethanol across the lipid membrane was recently determined by nuclear Overhauser enhancement spectroscopy and molecular dynamics simulations. ${ }^{6}$ It was concluded that the primary interaction between ethanol and the lipid bilayer occurs via formation of a hydrogen bond between the ethanol hydroxyl and the lipid phosphate and that the ethanol methyl is closer to the membrane center than its hydroxyl oxygen. ${ }^{6}$ In combination with our results, this indicates a relative orientation of ethanol and lipid headgroup as shown in Figure 3. The estimated lifetime of DMPC-ethanol association of a few nanoseconds obtained in this study is in excellent agreement with the lifetime estimated from the comparison of measured and calculated ethanol-lipid cross-relaxation rates that we reported earlier. ${ }^{6}$ Compared to the typical lifetime of association of a water molecule with the interface of phosphatidylcholine bilayers of $100 \mathrm{ps},{ }^{28,29}$ the time of association of ethanol molecules with a lipid bilayer is more than 1 order of magnitude longer. However, for most practical purposes free and bound ethanol are in very rapid exchange.

In our experiments at $14 \mathrm{wt} \%$ bicelles and physiological ethanol concentrations, about $4 \%$ of the ethanol was membranebound. This translates into about one bound ethanol molecule per 300 phosphatidylcholine molecules in the lipid matrix. While the number of bound ethanol molecules per lipid is rather low, ethanol binding to the lipids may nevertheless have a profound influence on the energetics of the lipid-water interface.

Acknowledgment. David Nizza is acknowledged for performing the gas chromatography measurements. We thank Ad 
Bax for the opportunity to record the solution NMR spectra in his lab and for stimulating discussions.

\section{References and Notes}

(1) Moxon, L. N.; Rose, S. E.; Haseler, L. J.; Galloway, G. J.; Brereton, I. M.; Bore, P.; Doddrell, D. M. Magn. Reson. Med. 1991, 19, 340-348.

(2) Smolen, T. N.; Smolen, A. Alcohol 1989, 6, 33-38.

(3) Spielman, D. M.; Glover, G. H.; Macovski, A.; Pfefferbaum, A. Alcohol, Clin. Exp. Res. 1993, 17, 1072-1077.

(4) Barry, J. A.; Gawrisch, K. Biochemistry 1994, 33, 8082-8088.

(5) Holte, L. L.; Gawrisch, K. Biochemistry 1997, 36, 4669-4674.

(6) Feller, S. E.; Brown, C. A.; Nizza, D. T.; Gawrisch, K. Biophys. J. 2002, 82, 1396-1404. 8905.

(7) Sanders, C. R.; Schwonek, J. P. Biochemistry 1992, 31, 8898-

(8) Nieh, M. P.; Glinka, C. J.; Krueger, S.; Prosser, R. S.; Katsaras, J. Langmuir 2001, 17, 2629-2638.

(9) Emsley, J. W.; Lindon, J. C. NMR spectroscopy using liquid crystal solvents; Pergamon Press: Oxford, 1975.

(10) Bax, A. Protein Sci. 2003, 12, 1-16.

(11) Ottiger, M.; Bax, A. J. Biomol. NMR 1998, 12, 361-372.

(12) Plateau, P.; Gueron, M. J. Am. Chem. Soc. 1982, 104, 7310-7311.

(13) Sklenar, V.; Bax, A. J. Magn. Reson. 1987, 74, 469-479.

(14) Davis, J. H.; Jeffrey, K. R.; Bloom, M.; Valic, M. I.; Higgs, T. P. Chem. Phys. Lett. 1976, 42, 390-394.
(15) Kadzhar, C. O.; Isaev, I. D.; Imanov, L. M. J. Struct. Chem. U.S.S.R 1968, 9, 375-377.

(16) Nakatsuji, H.; Morishima, I.; Kato, H.; Yonezawa, T. Bull. Chem. Soc. Jpn. 1971, 44, 2010-2017.

(17) Bryce, D. L.; Wasylishen, R. E. J. Biomol. NMR 2003, 25, 7378.

(18) Burnett, L. J.; Muller, B. H. J. Chem. Phys. 1971, 55, 5829-5831.

(19) Canet, D. NMR: Concepts and methods; Wiley: New York, 1996.

(20) Friebolin, H. Basic one- and two-dimensional NMR spectroscopy; VCH: New York, 1993.

(21) Sanders, C. R. Biophys. J. 1993, 64, 171-181.

(22) Barry, J. A.; Gawrisch, K. Biochemistry 1995, 34, 8852-8860.

(23) Kjellberg, A.; Homans, S. W. J. Magn. Reson. 2001, 151, 90-93.

(24) Struppe, J.; Komives, E. A.; Taylor, S. S.; Vold, R. R. Biochemistry 1998, 37, 15523-15527.

(25) Koenig, B. W.; Hu, J.-S.; Bose, S.; Hendler, R. W.; Bax, A. J. Am. Chem. Soc. 1999, 121, 1385-1386.

(26) Sass, J.; Cordier, F.; Hoffmann, A.; Rogowski, M.; Cousin, A.; Omichinski, J. G.; Löwen, H.; Grzesiek, S. J. Am. Chem. Soc. 1999, 121 , 2047-2055.

(27) Koenig, B. W.; Mitchell, D. C.; König, S.; Grzesiek, S.; Litman, B. J.; Bax, A. J. Biomol. NMR 2000, 16, 121-125.

(28) Volke, F.; Eisenblatter, S.; Galle, J.; Klose, G. Chem. Phys. Lipids 1994, 70, 121-131.

(29) Gaede, H. C.; Gawrisch, K. Magn. Reson. Chem. 2004, 42, 115- 